\title{
Diagnósticos de enfermagem ao idoso em instituição de longa permanência: revisão narrativa
}

\author{
Nursing diagnoses for the elderly in a long-term institution: a narrative review
}

\begin{abstract}
Diagnósticos de enfermería para adultos mayores en una institución de largo plazo: una revisión narrativa
\end{abstract}

Tânizi Lucateli Machado¹, Francine Casarin¹, Maria Helena Gehlen¹, Naiana Oliveira dos Santos', Silomar Ilha ${ }^{1 *}$.

\section{RESUMO}

Objetivo: Identificar, nas produções científicas, os principais diagnósticos de enfermagem no contexto da pessoa idosa em Instituição de Longa Permanência. Revisão bibliográfica: Identificaram-se 31 diagnósticos de enfermagem, quais sejam: atividade de recreação deficiente, nutrição desequilibrada: menor do que as necessidades corporais, deglutição prejudicada, eliminação urinária prejudicada, Incontinência urinária funcional, constipação, risco de constipação, troca de gases prejudicada, padrão de sono prejudicado, deambulação prejudicada, mobilidade física prejudicada, mobilidade física no leito prejudicada, mobilidade com cadeira de rodas prejudicada, confusão crônica, memória prejudicada, comunicação prejudicada, desesperança, baixa autoestima situacional, interação social prejudicada, ansiedade, tristeza crônica, risco de infecção, dentição prejudicada, risco de integridade da pele prejudicada, integridade da pele prejudicada, risco de quedas, dor aguda, dor crônica, isolamento social e risco de solidão. Tais diagnósticos, conforme a North American Nursing Diagnosis Association, representam 12 domínios: promoção da saúde, nutrição, eliminação e troca, atividade/repouso, percepção/cognição, autopercepção, papéis e relacionamentos, enfrentamento/ tolerância ao estresse, princípios da vida, segurança/ proteção e conforto. Considerações finais: Encontrou-se uma ampla quantidade/variedade de diagnósticos de enfermagem com idosos em Instituição de Longa Permanência na literatura, os quais foram comparados e discutidos com os dados de pesquisas realizadas em diferentes realidades, o que possibilitou perceber convergências, mesmo em contextos distintos.

Palavras-chave: Idoso, Instituição de longa permanência para idoso, Processo de enfermagem.

\begin{abstract}
Objective: To identify, in scientific productions, the main nursing diagnoses in the context of the elderly in a Long-Term Institution. Bibliographic review: Thirty-one nursing diagnoses were identified, namely: deficient recreational activity, unbalanced nutrition: less than the bodily needs, impaired swallowing, impaired urinary elimination, functional urinary incontinence, constipation, risk of constipation, impaired gas exchange, pattern of impaired sleep, impaired walking, impaired physical mobility, impaired physical mobility in bed, impaired wheelchair mobility, chronic confusion, impaired memory, impaired communication, hopelessness, low situational self-esteem, impaired social interaction, anxiety, chronic sadness, risk of infection, impaired dentition, risk of impaired skin integrity, impaired skin integrity, risk of falls, acute pain, chronic pain, social isolation and risk of loneliness. Such diagnoses, according to the North American Nursing Diagnosis
\end{abstract}

1 Universidade Franciscana (UFN), Santa Maria - RS. *E-mail: silo_sm@hotmail.com 
Association, represent 12 domains, they are: health promotion, nutrition, elimination and exchange, activity/rest, perception/cognition, self-perception, roles and relationships, coping/stress tolerance, principles of life, safety/protection and comfort. Final considerations: A large quantity/variety of nursing diagnoses with elderly in long-stay institutions was found in the literature, which were compared and discussed with data from surveys carried out in different realities, which made it possible to perceive convergences, even in different contexts.

Keywords: Aged, Homes for the aged, Nursing process.

\section{RESUMEN}

Objetivo: Identificar, en producciones científicas, los principales diagnósticos de enfermería en el contexto del adulto mayor en una Institución de Largo Plazo. Revisión bibliográfica: Se identificaron 31 diagnósticos de enfermería, a saber: actividad recreativa deficiente, nutrición desequilibrada: menos de las necesidades corporales, deglución deficiente, eliminación de orina alterada, incontinencia urinaria funcional, estreñimiento, riesgo de estreñimiento, intercambio de gases alterado, patrón de sueño alterado, alteración caminar, alteración de la movilidad física, alteración de la movilidad física en la cama, alteración de la movilidad en silla de ruedas, confusión crónica, alteración de la memoria, alteración de la comunicación, desesperanza, baja autoestima situacional, alteración de la interacción social, ansiedad, tristeza crónica, riesgo de infección, alteración de la dentición, riesgo de deterioro de la integridad de la piel, deterioro de la integridad de la piel, riesgo de caídas, dolor agudo, dolor crónico, aislamiento social y riesgo de soledad. Dichos diagnósticos, según la North American Nursing Diagnosis Association, representan 12 dominios: promoción de la salud, nutrición, eliminación e intercambio, actividad/descanso, percepción/cognición, autopercepción, roles y relaciones, afrontamiento/tolerancia al estrés, principios de vida, seguridad/protección y comodidad. Consideraciones finales: En la literatura se encontró una gran cantidad / variedad de diagnósticos de enfermería con ancianos en instituciones de larga estancia, los cuales fueron comparados y discutidos con datos de investigaciones realizadas en diferentes realidades, que permitieron percibir convergencias, incluso en diferentes contextos.

Palabras clave: Anciano, Hogares para ancianos, Proceso de enfermería.

\section{INTRODUÇÃO}

O envelhecimento populacional é o fenômeno complexo, com magnitude a nível mundial, o qual é definido como um processo natural, progressivo, irreversível, comum a todos os seres e que pode sofrer a influência de fatores sociais, políticos, econômicos e psicológicos. O número de idosos era de 202 milhões em 1950, passou para 1,1 bilhão em 2020 e deve alcançar 3,1 bilhões em 2100. No Brasil, em 2018, havia 63 idosos para cada 100 jovens e as projeções para 2055 estimam 202 idosos para cada 100 jovens (IBGE, 2018).

O envelhecimento está associado a um bom nível de saúde, contudo, à medida que as pessoas envelhecem, tornam-se mais susceptíveis as doenças crônicas não transmissível (DCNT) (MIRANDA GMD, et al., 2016). Tais condições conduzem, algumas vezes, a necessidades de cuidados constantes que demandam da família, como primeira unidade de cuidados, atenção, disponibilidade, recursos financeiros e/ou humanos para os quais, por vezes, não estão preparados (CASSOL AG, et al., 2018).

Frente a essa situação, emergem as Instituições de Longa Permanência para Idosos (ILPIs), as quais são definidas instituições públicas ou privadas, que servem de residências para pessoas com idade igual ou superior a 60 anos e que possuem ou não apoio e suporte da família. Como finalidade, essas instituições devem promover a liberdade, dignidade e a cidadania das pessoas idosas (BRASIL, 2005; ANVISA, 2020).

As ILPIS contam com a atuação de diferentes profissionais, dois quais se destaca os de enfermagem, pois são os que permanecem maior parte do tempo no cuidado direto/indireto as pessoas idosas institucionalizadas. Nesse contexto, tais profissionais, desenvolvem o cuidado com base na Sistematização 
da Assistência de Enfermagem (SAE), compreendida como uma metodologia que organiza o trabalho profissional de enfermagem quanto ao método pessoal e instrumentos, o que torna possível a operacionalização do processo de enfermagem (PE) (COFEN, 2009; SILVA MCN, 2017).

$\mathrm{O} P \mathrm{PE}$, por sua vez, caracteriza-se como um instrumento metodológico que orienta o profissional de enfermagem e a documentação da prática profissional, por meio de cinco etapas: Coleta de dados de enfermagem, diagnósticos, planejamento, implementação e avaliação de enfermagem (COFEN, 2009; SILVA MCN, 2017). Desde o ano de 2009, por meio da resolução de número 388, o Conselho Federal de Enfermagem (COFEN) torna obrigatório a implementação do PE de modo deliberado e sistemático, em todos os ambientes públicos ou privados, em que ocorre o cuidado profissional de enfermagem (COFEN, 2009; SANTOS MAP, DIAS PLM e GONZGA MFN, 2017).

Contudo, apesar da obrigatoriedade, percebe-se fragilidades na sua implementação, especialmente no que se refere aos diagnósticos de enfermagem que conduzem à implementação de cuidados, o que denota a necessidade de maiores estudos e investimentos para auxiliar nesse processo. Nesse sentido, torna-se relevante conhecer o que tem sido produzido na literatura sobre os diagnósticos de enfermagem e contextualizá-los com o contexto da pessoa idosa institucionalizada, fato que fundamenta a necessidade e relevância desse estudo. Justifica-se, ainda, pela compreensão de que as questões relativas à saúde da pessoa idosa são urgentes, sendo destacadas como prioridades de pesquisa no Brasil (BRASIL, 2018).

Frente ao exposto, questiona-se: quais os diagnósticos de enfermagem, mais utilizados nas produções científicas, no contexto da pessoa idosa institucionalizada? Na tentativa de responder o questionamento, objetivou-se identificar, nas produções científicas, os principais diagnósticos de enfermagem no contexto da pessoa idosa em Instituição de Longa Permanência.

\section{REVISÃO BIBLIOGRÁFICA}

Foram encontrados, inicialmente, um total de 42 estudos, os quais tiveram os seus títulos e resumos revisados. Essa etapa permitiu a inclusão de nove artigos. Os materiais selecionados foram submetidos a análise de conteúdo operacionalizada a partir de três etapas. Na primeira, realizou-se a leitura dos materiais e selecionou-se todos os diagnósticos de enfermagem encontrados nos estudos, os quais foram inseridos em uma tabela. Na segunda, explorou-se os diagnósticos de enfermagem elencados, buscando a sua identificação e confirmação da existência, bem como da sua correta descrição conforme a North American Nursing Diagnosis Association (NANDA, 2018). Esse momento oportunizou a exclusão dos diagnósticos com descrição incoerente ou aqueles que não foram encontrados na NANDA.

$\mathrm{Na}$ terceira etapa, realizou-se uma nova leitura dos diagnósticos buscando o agrupamento dos mesmos, ou seja, foram mantidos apenas aqueles identificados em dois ou mais estudos. Na sequência, os mesmos foram agrupados conforme o domínio ao qual faziam parte, bem como procedeu-se a sua definição, conforme a NANDA. A análise dos materiais permitiu a identificação de 31 diagnósticos de enfermagem à pessoa idosa institucionalizada, de acordo com o domínio, conforme pode ser visualizado no Quadro 1. 
Quadro 1 - Principais diagnósticos de enfermagem ao idoso institucionalizado, sua definição e domínio, Santa Maria - RS, 2020.

\begin{tabular}{|c|c|c|}
\hline Domínio da NANDA & Diagnósticos de enfermagem & Definição conforme NANDA \\
\hline 01- Promoção da Saúde & -Atividade de recreação deficiente. & $\begin{array}{l}\text { Estimulação (interesse ou envolvimento) diminuída em atividades recreativas ou de } \\
\text { laser. }\end{array}$ \\
\hline \multirow{2}{*}{ 02- Nutrição } & $\begin{array}{l}\text { - Nutrição desequilibrada: menor do que as } \\
\text { necessidades corporais. }\end{array}$ & Ingestão insuficiente de nutrientes para satisfazer as necessidades metabólicas. \\
\hline & - Deglutição prejudicada. & $\begin{array}{l}\text { Funcionamento anormal do mecanismo da deglutição associado a déficits na estrutura } \\
\text { ou função oral, faríngea ou esofágica. }\end{array}$ \\
\hline \multirow{5}{*}{ 03- Eliminação e Troca } & - Eliminação urinária prejudicada. & Disfunção na eliminação da urina. \\
\hline & - Incontinência urinária funcional. & $\begin{array}{l}\text { Incapacidade da pessoa que é geralmente continente de alcançar o banheiro a tempo } \\
\text { de evitar a perda não intencional de urina. }\end{array}$ \\
\hline & - Constipação. & $\begin{array}{l}\text { Diminuição na frequência normal de evacuação, acompanhada de eliminação difícil } \\
\text { ou incompleta de fezes e/ou eliminação de fezes excessivamente difíceis e duras. }\end{array}$ \\
\hline & - Risco de constipação. & $\begin{array}{l}\text { Diminuição na frequência normal de evacuação, acompanhada de eliminação difícil } \\
\text { ou incompleta de fezes e/ou eliminação de fezes excessivamente difíceis e duras. }\end{array}$ \\
\hline & - Troca de gases prejudicada. & $\begin{array}{l}\text { Excesso ou déficit na oxigenação e/ou eliminação de dióxido de carbono na membrana } \\
\text { alvéolo capilar. }\end{array}$ \\
\hline \multirow{5}{*}{ 04- Atividade/ Repouso } & - Padrão de sono prejudicado. & $\begin{array}{l}\text { Interrupções, limitadas pelo tempo, da quantidade e da qualidade do sono, } \\
\text { decorrentes de fatores externos. }\end{array}$ \\
\hline & - Deambulação prejudicada. & Limitação do movimento independente de andar em um determinado ambiente. \\
\hline & - Mobilidade física prejudicada. & $\begin{array}{l}\text { Limitação de movimento físico independente e voluntário do corpo ou de uma ou mais } \\
\text { extremidades. }\end{array}$ \\
\hline & -Mobilidade física no leito prejudicada. & Limitação do movimento independente de uma posição para outra no leito. \\
\hline & - Mobilidade com cadeira de rodas prejudicada. & Limitação de operação independente com cadeira de rodas pelo ambiente. \\
\hline \multirow[t]{3}{*}{ 05- Percepção/ Cognição } & - Confusão crônica. & $\begin{array}{l}\text { Deterioração irreversível, prolongada e/ou progressiva do intelecto e da } \\
\text { personalidade, definida por capacidade diminuída para interpretação dos estímulos } \\
\text { ambientais e para processos de pensamento intelectual, manifestada por distúrbios } \\
\text { de memória, orientação e comportamento. }\end{array}$ \\
\hline & - Memória prejudicada. & Incapacidade de recordar ou recuperar informações ou habilidades comportamentais \\
\hline & - Comunicação prejudicada. & $\begin{array}{l}\text { Capacidade diminuída, retardada ou ausente para receber, processar, transmitir e/ou } \\
\text { usar um sistema de símbolos. }\end{array}$ \\
\hline \multirow{2}{*}{ 06- Autopercepção } & - Desesperança. & $\begin{array}{l}\text { Estado subjetivo no qual o indivíduo vê alternativas limitadas ou não vê alternativas } \\
\text { ou escolhas pessoais disponíveis e é incapaz de mobilizar energias a seu favor. }\end{array}$ \\
\hline & - Baixa autoestima situacional. & $\begin{array}{l}\text { Desenvolvimento de percepção negativa sobre o seu próprio valor em resposta a uma } \\
\text { situação atual. }\end{array}$ \\
\hline
\end{tabular}




\begin{tabular}{|c|c|c|}
\hline Domínio da NANDA & Diagnósticos de enfermagem & Definição conforme NANDA \\
\hline 07- Papéis e Relacionamentos & - Interação social prejudicada. & Quantidade insuficiente ou excessiva, ou qualidade ineficaz, de troca social. \\
\hline \multirow{2}{*}{$\begin{array}{c}\text { 09- Enfrentamento/ Tolerância } \\
\text { ao estresse }\end{array}$} & - Ansiedade. & $\begin{array}{l}\text { Incômodo sentimento do desconforto ou temor, acompanhado por resposta } \\
\text { autonômica (a fonte é frequentemente não específica ou desconhecida para o } \\
\text { indivíduo), sentimento de apreensão causada pela antecipação de perigo. É um sinal } \\
\text { de alerta que chama a atenção para um perigo iminente e permite ao indivíduo tomar } \\
\text { medidas para lidar com a ameaça. }\end{array}$ \\
\hline & - Tristeza crônica. & $\begin{array}{l}\text { Padrão clínico, recorrente e potencialmente progressivo de tristeza disseminada, } \\
\text { vivenciada (por pai/ mãe, cuidador, indivíduo com enfermidade crônica ou deficiência) } \\
\text { em resposta à perda contínua ao longo da trajetória de uma enfermidade ou } \\
\text { deficiência. }\end{array}$ \\
\hline 10- Princípios da vida & - Disposição para bem-estar espiritual melhorado. & $\begin{array}{l}\text { Padrão de confiança em crenças religiosas e/ ou participação em rituais de uma fé } \\
\text { religiosa em particular, que pode ser fortalecida. }\end{array}$ \\
\hline \multirow{5}{*}{ 11- Segurança/ Proteção } & - Risco de infecção. & $\begin{array}{l}\text { Vulnerabilidade à invasão e multiplicação de organismos patogênicos, que pode } \\
\text { comprometer a saúde. }\end{array}$ \\
\hline & - Dentição prejudicada. & $\begin{array}{l}\text { Ruptura nos padrões de desenvolvimento/erupção dentários ou na integridade } \\
\text { estrutural de cada dente. }\end{array}$ \\
\hline & - Risco de integridade da pele prejudicada. & $\begin{array}{l}\text { Risco de epiderme e/ou derme alterada, que pode comprometer a saúde ou no risco } \\
\text { para que isso ocorra. }\end{array}$ \\
\hline & - Integridade da pele prejudicada. & $\begin{array}{l}\text { Epiderme e/ou derme alterada, que pode comprometer a saúde ou no risco para que } \\
\text { isso ocorra. }\end{array}$ \\
\hline & - Risco de quedas. & $\begin{array}{l}\text { Vulnerabilidade e aumento da suscetibilidade a quedas, que pode causar dano físico } \\
\text { e comprometer a saúde. }\end{array}$ \\
\hline \multirow{4}{*}{ 12- Conforto } & - Dor aguda. & $\begin{array}{l}\text { Experiência sensorial e emocional desagradável associada à lesão tissular real ou } \\
\text { potencial, ou descrita em termos de tal lesão, início súbito ou lento, de intensidade } \\
\text { leve a intensa, com término antecipado ou previsível. }\end{array}$ \\
\hline & - Dor crônica. & $\begin{array}{l}\text { Experiência sensorial e emocional desagradável associada à lesão tissular real ou } \\
\text { potencial, ou descrita em termos de tal lesão, início súbito ou lento, de intensidade } \\
\text { leve a intensa, constante ou recorrente, sem término antecipado ou previsível e com } \\
\text { duração maior que três meses. }\end{array}$ \\
\hline & - Isolamento social. & $\begin{array}{l}\text { Solidão experienciada pelo indivíduo ou percebida como imposta por outros e como } \\
\text { um estado negativo ou ameaçador. }\end{array}$ \\
\hline & - Risco de solidão. & $\begin{array}{l}\text { Vulnerabilidade a experienciar desconforto associado a desejo ou necessidade de } \\
\text { mais contato com os outros, que pode comprometer a saúde. }\end{array}$ \\
\hline
\end{tabular}

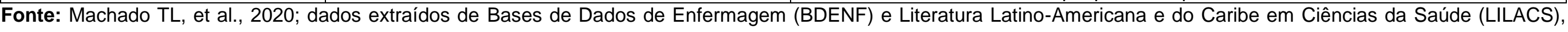
Google acadêmico e confirmados na NANDA. 
No domínio promoção da saúde, destacou-se nos estudos analisados, o diagnóstico de enfermagem: atividade de recreação deficiente. Estudo desenvolvido com pessoas idosas institucionalizadas com objetivo de identificar as atividades de lazer realizadas em ILPI, demonstrou que $31 \%$ das instituições não oferecia atividades de lazer, ou não eram adequadas para as condições de saúde do idoso, ou ainda, que as pessoas idosas não sentiam vontade de realizá-las (CASTRO VC e CARREIRA L, 2015).

Estudo que objetivou compreender a percepção do idoso institucionalizado sobre os benefícios das oficinas terapêuticas demonstrou que os participantes reconhecem a associação entre a prática das atividades terapêuticas e melhoria de qualidade de vida (SILVA TG, et al., 2016). Assim, compreende-se a necessidade de estimular a prática de atividades recreativas e de laser junto as pessoas idosas institucionalizadas, uma vez que auxiliam no bem-estar e na qualidade de vida.

No domínio Nutrição, evidenciaram-se dois diagnósticos: nutrição desequilibrada: menor do que as necessidades corporais e deglutição prejudicada. A alimentação é fundamental para a nutrição e para a sobrevivência. Contudo, existem alguns fatores que podem interferir na nutrição desequilibrada dos idosos, dentre eles, aspectos biológicos, psicológicos e/ou econômicos, como também a presença de patologias que podem alterar os processos metabólicos (FERREIRA CJ, et al., 2010). A degeneração fisiológica do mecanismo da deglutição, como resultado da senescência, ocorre em todas as fases da deglutição, devido às mudanças estruturais, anatômicas e funcionais interferindo na deglutição da pessoa idosa saudável (BASTOS LS, et al., 2016).

Nesse contexto, torna-se importante o conhecimento da anatomia da cavidade oral, faringe, laringe e esôfago, pelo qual é transportado o bolo alimentar até o estômago para que o mecanismo anatômico e fisiológico da deglutição possa ser atentamente acompanhado e orientado (BASTOS LS, et al., 2016). Qualquer interferência ou dificuldade no processo de deglutição é denominado de disfagia e merece ações de cuidado específicas para prevenção e/ou reabilitação dessa condição (BASTOS LS, et al., 2016).

No domínio eliminação e troca, destacaram-se cinco diagnósticos: eliminação urinária prejudicada; incontinência urinária funcional; risco de constipação; constipação e troca de gases prejudicada. Estudo desenvolvido com 12 pessoas idosas em um ambulatório de referência em Teresina no estado do Piauí, demonstrou que a incontinência urinária nos idosos é uma patologia pouco discutida e que a baixa escolaridade dos mesmos, influencia na demora em procurar o tratamento e esclarecimento sobre a doença. Além disso, tal situação produz sentimentos negativos nos idosos, como ansiedade, receio, preocupação, baixa autoestima e frustração, culminando com a exclusão do convívio social, pois passam a se preocupar com a disponibilidade de banheiro, envergonham-se com o odor da urina e passam a segurá-la (MATOS MAB, et al., 2019).

A constipação intestinal tem uma prevalência entre 10 a 15\% em países desenvolvidos e pode se correlacionar a vários fatores como: idade, condições ambientais, dietas, genética, fatores socioeconômicos, saúde pública, diversidade cultural, podendo ser avaliada pela classificação de critérios de avaliação da constipação (PICH PC, et al., 2013).

Sobre a constipação em idosos, sabe-se que alguns fatores predispõem essa complicação, dentre eles, o baixo consumo de fibras e água, eliminação fecal errônea, ausência de atividades físicas e a perda do tônus da musculatura intestinal. Estudo desenvolvido com o objetivo de verificar a prevalência de diagnósticos da constipação intestinal e suas causas multifatoriais entre idosos de uma ILPI do estado de Goiás, demonstrou que o consumo de frutas e legumes juntamente com o consumo hídrico é fundamental para auxiliar a reduzir o risco de constipar (SANTOS CRS e BARBOSA LBG, 2017).

Para tanto o diagnóstico da constipação é essencial para uma conduta dietética adequada; o consumo de verduras e frutas são essenciais e o aumento hídrico ajuda a ter uma eliminação fecal satisfatória e sem dor. A atividade física, por sua vez, auxilia na formação e na eliminação das fezes (SANTOS CRS e BARBOSA LBG, 2017).

No diagnóstico de enfermagem, troca de gases prejudicada é indispensável a realização de uma análise profunda das necessidades básicas afetadas pelo paciente, ajustando qualquer atividade básica para uma 
assistência planejada, crítica e científica. A identificação do diagnóstico está mais presente em pessoas com alterações cardíacas e pode assinalar a presença de distúrbios importantes, dada sua íntima relação com o sistema cardiovascular (PASCOAL LM, et al., 2009).

No domínio atividade/repouso, identificaram-se cinco diagnósticos: padrão de sono prejudicado; deambulação prejudicada; mobilidade física prejudicada; mobilidade física no leito prejudicada e mobilidade com cadeira de rodas prejudicada. Com o envelhecimento existe redução da eficiência do sono, sendo frequente ocorrerem diversas alterações que contribuem para o aumento de vezes em que a pessoa idosa acorda durante a noite, dentre elas se destaca a dificuldade em manter o sono e a ausência de um sono reparador, o que contribui para a sonolência diurna. Para além destas observa-se, também, a redução do sono em profundidade, bem como a alteração do ciclo do sono-vigília, o que conduz ao aumento de vezes em que o idoso se acorda durante a noite. Tais alterações, podem contribuir para o déficit de cognição e aumento dos riscos de desenvolvimento de doenças demenciais, fato que alerta à importância das horas dormidas na manutenção das funções cognitivas na pessoa idosa (YAFFE K, et al., 2014).

No que concerne à deambulação prejudicada, estudo desenvolvido com 191 idosos demonstrou elevadas as taxas de impossibilidade em andar e a utilização de órteses. Quanto a mobilidade física, mobilidade no leito e na cadeira de rodas prejudicada, o estudo demonstrou uma prevalência de dificuldade para locomoção de $50,3 \%$. Quanto ao mecanismo acessório para a marcha, $41,7 \%$ utilizavam cadeira de rodas, $24,0 \%$ eram acamados (tinham momentos na cadeira de rodas, mas incapazes de realizarem as transferências), 16,7\% faziam uso do andador, $14,6 \%$ bengala e 3,1\% muletas (GIAQUINI F, et al., 2017).

Nesse sentido, salienta-se a relevância dos diagnósticos de enfermagem sobre a mobilidade em pessoas idosas, pois a partir deles podem ser implementadas medidas com vistas à minimização das complicações inerentes às dependências para deambular. Assim, compreende-se que a promoção da saúde, bem como as ações preventivas a fim de evitar ou retardar a dificuldade de locomoção, com a máxima manutenção das habilidades locomotoras devem ser trabalhadas. Para as pessoas com quadros mais graves, cadeirantes e acamados, o foco são os cuidados com as complicações decorrentes da imobilização, bem como o estimulo muscular frequente (GIAQUINI F, et al., 2017).

No domínio percepção/cognição, identificaram-se três diagnósticos: confusão crônica; memória prejudicada e comunicação verbal prejudicada. A confusão crônica está relacionada com a deterioração irreversível, prolongada e/ou progressiva do intelecto e da personalidade (SOUZA RM, et al., 2010). A identificação desta alteração se faz necessária para prevenção do seu agravamento e complicações futuras. Estudo desenvolvido com 61 idosos, objetivando analisar o diagnóstico de enfermagem Memória Prejudicada, demonstrou como riscos potenciais, as quedas, infecções, incontinência, úlceras por pressão, desidratação, delírio, imobilidade, depressão, os quais podem interferir diretamente no prejuízo da memória, assim como o prejuízo da memória pode agravar esses riscos. Assim, é necessário que o enfermeiro seja capaz de identificar a causa das complicações que podem ser apresentadas, como problemas secundários às causas primárias, com vistas a promover intervenções para auxiliar nesse contexto (SOUZA PA e SANTANA RF, 2011).

Revisão integrativa que analisou 20 estudos, identificou a utilização do diagnóstico de enfermagem comunicação verbal prejudicada, onde doze $(60 \%)$ dos estudos relataram alta prevalência $(75-100 \%)$ do diagnóstico em distintos contextos clínicos, geralmente associado ao impedimento verbal por barreiras físicas, tais como: intubação/traqueostomia e alterações neurológicas. Contudo, os autores referem que o diagnóstico Comunicação Verbal Prejudicada tem sido utilizado na prática clínica de maneira superficial, sem uma avaliação precisa e detalhada do prejuízo na comunicação (PUGGINA ACG, et al., 2016).

No domínio autopercepção, identificaram-se dois diagnósticos: desesperança e baixa autoestima situacional. A desesperança, por vezes, pode estar associada a transtornos depressivos, uma vez que as pessoas que apresentam depressão tendem a avaliar a si mesmas, o mundo e o futuro de forma negativa. Além disso, sentimentos característicos do envelhecer podem despertar a desesperança e conduzir a sintomas depressivos e ansiosos, pois muitas vezes, as pessoas idosas deixam de fazer atividades que não 
conseguiram, em algum momento realizar, pois sentem receio de novamente não conseguir (OLIVEIRA KL, et al., 2006). Revisão de literatura demonstrou associação entre a baixa autoestima e a expressão de tristeza, ansiedade, desanimo, isolamento, temor, impotência, dores diversas, expressão de incapacidades, negação, ruminação de problemas, autoacusação, comportamentos hostis consigo mesmo e com os outros, além da diminuição do interesse e motivação para os cuidados com a própria higiene (DIAS CA, et al., 2011).

No domínio papéis e relacionamentos, identificou-se um diagnóstico: interação social prejudicada. Esse diagnóstico possui relação com o envelhecimento, uma vez que essa fase da vida é frequentemente sentida, por algumas pessoas idosas, como uma descontinuidade do que já viveram, já que o desejo e o interesse pelo convívio social assíduo não apresentam a mesma intensidade do passado. Além disso, pensar nos idosos como uma categoria de pessoas a parte, conduz a compreensão dos mesmos como pessoas desligadas da vida em sociedade naturalmente construída ao longo da vida, bem como sobre tudo que a relação da pessoa idosa com a sociedade representa (MARTINS R, et al., 2010).

No domínio enfrentamento/tolerância ao estresse, identificaram-se dois diagnósticos: ansiedade e tristeza crônica. A ansiedade, caracteriza-se como uma situação gerada a partir da visualização dos eventos como algo caótico, como se alguma coisa perigosa e, portanto, ameaçadora estivesse por acontecer (OLIVEIRA $\mathrm{KL}$, et al., 2006). No processo de institucionalização, a pessoa idosa se depara com alguns fatores que influenciam no seu estado mental, como a troca de ambiente e a convivência com pessoas desconhecidas. Assim, a institucionalização pode causar ansiedade e depressão, pelo medo de serem esquecidas, rejeitadas e desamparadas pela família e pela sociedade (CARMO HO, et al., 2012). No que se refere a tristeza, estudo epidemiológico transversal realizado com 673 idosos, com objetivo de identificar os sentimentos dos mesmos sobre a vida, o futuro e as necessidades por eles sentidas, em função do local de residência, demonstrou que $63,2 \%$ das pessoas idosas confirmaram sentir-se triste (MARTINS R, et al., 2010).

No domínio princípios da vida, identificou-se um diagnóstico: disposição para bem-estar espiritual melhorado. Esse diagnóstico demonstra-se relevante, uma vez que as pessoas idosas pelos anos vividos, naturalmente, tiveram perdas. Quanto mais o ser humano envelhece, maiores são as perdas, as quais somam-se e podem conduzir a consequências negativas em sua saúde. Nesse sentido, tanto a religião, quanto a espiritualidade podem ser ferramentas de auxílio ao enfrentamento das situações consideradas estressoras (DUARTE FM e WANDERLEY KS, 2011).

No domínio segurança/proteção, identificaram-se cinco diagnósticos: risco de infecção; dentição prejudicada; risco de integridade da pele prejudicada; integridade da pele prejudicada, e risco de quedas. As alterações do sistema imunológico da pessoa idosa, a torna mais suscetíveis às infecções como a pneumonia, infecção urinária, infecção de sitio cirúrgico e sepse, as quais são as mais comuns nesta população. Tais infeç̧ões, quando evidenciadas na pessoa idosa, são mais graves e mais difíceis de tratar do que em adultos, colaborando para elevação dos indicadores de mortalidade nessa população (BERTOL CD, et al., 2020). No que diz respeito a dentição prejudicada, a perda dentária é considerada, no Brasil, um problema de saúde pública especialmente vivenciado pelas pessoas idosas. Assim, deve ser conduzido por políticas de saúde bucal com vistas a redução dos danos gerados por essa situação (TEIXEIRA CM, et al.; 2016).

No que se refere as questões relativas à pele da pessoa idosa, com o processo de envelhecimento o turgor e elasticidade da pele diminuem. Assim, é fundamental que o enfermeiro avalie o turgor e nível de hidratação da pele dos idosos para que se possa prescrever as intervenções de enfermagem específicas de acordo com as necessidades de cada pessoa, a partir do seu tipo de pele (GIARETTA VMA, et al., 2016). Quanto ao risco de quedas, pesquisa demonstrou que $71,4 \%$ das pessoas idosas avaliadas sofreram de uma a duas quedas e, como causas intrínsecas, se identificou a tontura/vertigem; e como extrínseca, os pisos escorregadios ou molhados. Portanto, é relevante avaliar o risco de quedas em pessoas idosas para que medidas preventivas sejam tomadas com o objetivo de maximizar a qualidade de vida (PINHO TAM, et al., 2012).

No domínio conforto, identificaram-se quatro diagnósticos: dor aguda; dor crônica; isolamento social e risco de solidão. A população idosa pode torna-se mais susceptível ao desenvolvimento de dor crônica uma vez que estão mais expostos a traumatismos, infecções e doenças crônico-degenerativas, responsáveis por esse 
tipo de dor. Além disso, a dor está entre os principais fatores que limitam a possibilidade da pessoa idosa em manter seu cotidiano habitual, impactando negativamente sua qualidade de vida, prejudicando as atividades de vida diária (AVDs), e restringindo a convivência social, o que pode conduzir ao isolamento (CELIH VC e GALON C, 2009).

Esse dado revela o maior risco do idoso para o isolamento social e solidão. A esse respeito, estudo desenvolvido com idosas que objetivou investigar o sentimento de solidão, bem como o papel da rede de apoio social na vivência dessa fase do ciclo vital, demonstrou que as pessoas idosas reconhecem que o sentimento de solidão pode aumentar com a idade. Além disso, que as pessoas idosas referem que a manutenção de vínculos e atividades sociais se constituiu como um fator protetivo em relação à solidão (CARMONA CF, et al., 2014).

\section{CONSIDERAÇÕES FINAIS}

Destacaram-se 31 diagnósticos de enfermagem, os quais corresponderam a 11 domínios do NANDA: promoção da saúde; nutrição; eliminação e troca; atividade/repouso; percepção/cognição; auto percepção; papéis e relacionamentos; enfrentamento/tolerância ao estresse; princípios da vida; segurança/proteção; e conforto. Como contribuições desse estudo, entende-se que o mesmo pode ser utilizado como base para outras pesquisas acerca da temática, bem como para repensar as questões relativas ao PE à pessoa idosa em diferentes contextos o que contribuirá para o cuidado e saúde da pessoa idosa. Outrossim, esperasse que possa estimular os profissionais de enfermagem para construção de novas propostas de pesquisa e, que essas, reflitam no melhor cuidado ao idoso em diferentes cenários e contextos de saúde.

\section{REFERÊNCIAS}

1. ANVISA. Agência Nacional de Vigilância Sanitária. Instituições de Longa Permanência para Idosos (ILPIs). Brasília (DF): Ministério da saúde; 2020. Disponível em: https://www.gov.br/anvisa/ptbr/assuntos/servicosdesaude/instituicoes-de-longa-permanencia-para-idosos

2. BASTOS LS, et al. Disfagia em idosos e os agenciamentos corporal/emocional: reflexões teórico-prática sobre como estamos tratando. Revista práxis, 2016; 8(15): 43-15.

3. BERTOL CD, et al. Avaliação das infecções Hospitalares em idosos. Revista Interdisciplinar de Estudos em Saúde da UNIARP. 2020; 1(19): 1-10.

4. BRASIL. Ministério da Saúde (BR). Resolução da Diretoria Colegiada - RDC/ ANVISA No 283, de 26 de setembro de 2005. Brasília (DF): Ministério da Saúde; 2005. Disponível em: http://bvsms.saude.gov.br/bvs/saudelegis/anvisa/2005/res0283_26_09_2005.html. Acessado em: 17 mar. 2021.

5. BRASIL. Ministério da Saúde. Agenda nacional de prioridades de pesquisa em saúde. Brasília, DF:MS; 2018. Disponível em: http:// bvsms.saude.gov.br/bvs/publicacoes/agenda_prioridades_pesquisa_ms.pdf. Acesso em 20 de dez. 2020.

6. CARMO HO, et al. Idoso Institucionalizado: O que sente, percebe e deseja? RBCEH, Passo Fundo, 2012; 9(3): 330340.

7. CARMONA CF, et al. A experiência de solidão e a rede de apoio social de idosas. Psicologia em Estudo, Maringá, 2014; 19(4): 681-691.

8. CASSOL AG, et al. (Re)Organização familiar de pessoas idosas com a doença de Alzheimer na perspectiva da complexidade. Disciplinarum Scientia. Série: Ciências da Saúde, 2018, 19(1): 11-23.

9. CASTRO VC, CARREIRA L. Atividades de lazer e atitude de idosos institucionalizados: subsídios para a prática de enfermagem. Rev. Latino-Am. Enfermagem, 2015; 23(2): 307-314.

10. CELICH KLS, GALON C. Dor crônica em idosos e sua influência nas atividades da vida diária e convivência social. Rev. bras. geriatr. gerontol. 2009; 12(3): 345-359.

11. CONSELHO FEDERAL DE ENFERMAGEM (COFEN). Resolução COFEN-358/2009. Brasília-DF, 2009. Disponível em: http://www.cofen.gov.br/resoluo-cofen-3582009_4384.html.

12. DIAS CA, et al. O enfermeiro na identificação do diagnóstico de enfermagem baixa autoestima, na assistência a idosos com transtorno afetivo. Revista Científica da Faculdade de Educação e Meio Ambiente, 2011; 2(Supl-I): 89-91.

13. DUARTE FM, WANDERLEY KS. Religião e Espiritualidade de Idosos Internados em uma Enfermaria Geriátrica. Psicologia: Teoria e Pesquisa, 2011; 27(1): 49-53. 
14. FERREIRA CJ, et al. Diagnósticos de enfermagem identificados em idosos assistidos na atenção básica no Brasil. Rev Enferm UFPE Online, 2010; 4(Esp 1): 962-971.

15. GIAQUINI F, et al. Prevalência de dificuldade de locomoção em idosos institucionalizados. Acta Fisiatr., 2017; 24(1): 1-6.

16. GIARETTA VMA, et al. Proposta de escala para avaliar o turgor da pele de idosos. Rev Ciên Saúde, 2016; 1(1): 0107.

17. INSTITUTO BRASILEIRO DE GEOGRAFIA E ESTATÍSTICA (IBGE). Tabela2010-2060-Projeção daPopulação (revisão 2018), Rio de Janeiro: IBGE, 2018. Disponível em: https://agenciadenoticias.ibge.gov.br/agencia-sala-de-imprensa/2013agencia-de-noticias/releases/21837-projecao-da-populacao-2018-numero-de-habitantes-do-pais-deve-parar-decrescer-em-2047. Acesso em: 08 mar. 2021.

18. MARTINS R, et al. A vida vista pelos idosos. Millenium, 2010; 39: 121- 130.

19. MATOS MAB, et al. As Repercussões Causadas pela Incontinência Urinária na Qualidade de Vida do Idoso. Rev Fund Care Online, 2019; 11(3): 567-575.

20. MIRANDA GMD, et al. O envelhecimento populacional brasileiro: desafios e consequências sociais atuais e futuras. Rev. Bras. Geriatr. Gerontol. Rio de Janeiro, 2016; 19(3): 507-519.

21. NANDA. Diagnósticos de enfermagem da NANDA. Definições e classificações, 11. ed. Porto Alegre: Artmed, 2018. 462p.

22. OLIVEIRA KL, et al. Relação entre ansiedade, depressão e desesperança entre grupos de idosos. Psicologia em Estudo, Maringá, 2006; 11(2): 351-359.

23. PASCOAL LM, et al. Indicadores de troca de gases prejudicada em pacientes com doenças cardiovasculares segundo a NOC. Rev. RENE, 2009; 10(4): 96-102.

24. PICH PC, et al. Avaliação do trânsito intestinal em relação ao estado de vida em idosos de um clube de terceira idade. Unopar cient ciênc biol saúde, 2013; 15(3): 207-213.

25. PINHO TAM, et al. Avaliação do risco de quedas em idosos atendidos em Unidade Básica de Saúde. Rev. esc. Enferm. USP. 2012; 46(2): 320-327.

26. PUGGINA ACG, et al. Nursing diagnosis impaired verbal communication in clinical practice: an integrative review. Revista Família Ciclos de Vida e Saúde no Contexto Social -REFACS, 2016; 4(2): 135-144.

27. SANTOS CRS, BARBOSA LBG. Constipação intestinal, diagnóstico e causa multifatorial em idosos de instituição de longa permanência. Rev. Cient. Sena Aires, 2017; 6(2): 95-102.

28. SANTOS MAP, DIAS PLM, GONZGA MFN. Processo de enfermagem sistematização da assistência de enfermagem -SAE. Revista Saúde em Foco. 2017; 1(9): 679-683.

29. SILVA MCN. Sistematização da assistência de Enfermagem: desafio para a prática profissional. Enferm Foco. 2017; $8(3)$.

30. SOUSA RM, et al. Diagnósticos de enfermagem identificados em idosos hospitalizados: associação com as síndromes geriátricas. Escola Anna Nery, 2010; 14(4): 732-741.

31. SILVA MR, et al. A percepção do idoso institucionalizado sobre os benefícios das oficinas terapêuticas. Rev Bras Promoção Saúde, Fortaleza, 2016; 29(Supl): 76-84.

32. SOUSA RM, et al. Diagnósticos de enfermagem identificados em idosos hospitalizados: associação com as síndromes geriátricas. Escola Anna Nery, 2010; 14(4): 732-741.

33. SOUZA PA, SANTANA RF. The nursing diagnosis, impaired memory, in hospitalized elderly. Acta Paul Enferm., 2011; 24(1): 36-42.

34. TEIXEIRA CM, et al. Atividade física, autoestima e depressão em idosos. Cuadernos de Psicologia del deporte. 2016; 16(3): 55-66.

35. YAFFE K, et al. Connections between sleep and cognition in older adults. Lancet Neurol., 2014; 13(10): 1017-1028. 